\title{
The Effectiveness and Value of Deflazacort and Exon-Skipping Therapies for the Management of Duchenne Muscular Dystrophy
}

\author{
A Summary from the Institute for Clinical and Economic Review's \\ New England Comparative Effectiveness Public Advisory Council
}

Foluso Agboola, MBBS, MPH; Grace A. Lin, MD, MAS; Noemi Fluetsch, MPH; Surrey M. Walton, PhD; David M. Rind, MD, MSc; and Steven D. Pearson, MD, MSc

$\mathrm{D}$ uchenne muscular dystrophy (DMD) is a fatal, X-linked neuromuscular disease characterized by progressive loss of muscle function due to the loss of expression of the dystrophin protein, typically to less than $3 \%$ of normal. ${ }^{1}$ Although rare, it is the most common pediatric muscular dystrophy, with a prevalence of 1 in 3,500-5,000 live male births or about 400-600 per year in the United States. ${ }^{2}$ The mean age of onset of symptoms is 2.5 years; however, diagnosis is often delayed until around age $5 .^{3}$

The initial symptoms of DMD include muscle weakness, clumsiness, and difficulty going up and down stairs, and untreated children with DMD usually progress to a loss of ambulation by age $10{ }^{4}$ Patients may also have cognitive and behavioral issues, impaired growth, and gastrointestinal or orthopedic complications. Because of the significant disability caused by DMD, quality of life is diminished, and caregiving burden is high. Patients commonly develop fatal respiratory or cardiac complications in the second or third decade of life, with many deaths occurring following surgery or in the setting of an acute infection such as pneumonia.,

Standard treatment for DMD includes supportive care, such as physical and occupational therapy to maintain ambulation, and medications such as corticosteroids. Corticosteroids, including prednisone and deflazacort (Emflaza, PTC Therapeutics), are the mainstay of drug therapy for DMD. While prednisone does not have a U.S. Food and Drug Administration (FDA) indication specific for DMD, it is widely used. Deflazacort was first licensed for use overseas in the 1980s and was approved in February 2017 for treatment of DMD in the United States.

Exon-skipping therapies are a novel class of drugs that skip mutated exons of the DMD gene, thereby resulting in production of some amount of shortened dystrophin that could be beneficial in slowing progression of the disease for patients with certain mutations. Eteplirsen (Exondys 51, Sarepta Therapeutics) was the first exon-skipping therapy to be approved by the FDA; it was approved in September 2016 for DMD patients with mutations amenable to skipping of exon 51 (estimated to be approximately 13\% of the DMD population).

J Manag Care Spec Pharm. 2020;26(4):361-66

Copyright $\odot 2020$, Academy of Managed Care Pharmacy. All rights reserved.
Golodirsen (Vyondys 53, Sarepta Therapeutics) was approved by the FDA on December 12, 2019, for patients with mutations amenable to exon 53 skipping (estimated to be $9 \%$ of the DMD population). These drugs represent the first targeted treatments for DMD.

The Institute for Clinical and Economic Review (ICER) performed a systematic literature review and cost-effectiveness analysis to evaluate the health and economic outcomes of corticosteroids and exon-skipping therapies as treatments for DMD. Complete details of ICER's systematic literature search and protocol, as well as the methodology and model structure for the economic evaluation, are available on ICER's website (www. icer-review.org). In this article, we present the summary of our findings and highlights of the policy discussion with key stakeholders regarding the overall value of these therapies held at a public meeting of the New England Comparative Effectiveness Public Advisory Council on July 25, 2019. ${ }^{7}$ The detailed report is available on the ICER website at https://icer-review.org/material/dmd-final-evidence-report/.

\section{Summary of Findings}

\section{Clinical Effectiveness}

Our review was framed to evaluate 2 comparisons: (1) deflazacort versus prednisone and (2) eteplirsen and golodirsen versus supportive care.

Deflazacort Versus Prednisone. We identified 3 randomized controlled trials (RCTs) of varying quality that assessed the efficacy of deflazacort compared with prednisone. Due mainly to differences in study design and outcomes measured and reported, we summarized but did not quantitatively synthesize the evidence through meta-analysis. The largest RCT, which was rated as good quality, was a multicenter, phase 3 trial conducted in 196 boys (aged 5-15 years) with DMD. ${ }^{8}$ The 2 other RCTs were smaller studies of fair or poor quality.,10 Measures of clinical benefit assessed in one or more of the trials include motor function, muscle strength, and pulmonary function. Results on motor function were mixed, with 2 of the RCTs showing statistically significant improvement favoring deflazacort at 12 months for some outcomes (time to climb 4 stairs, composite motor function outcome), ${ }^{8,9}$ while 1 RCT found no statistically significant difference in motor 
function between deflazacort-and prednisone-treated patients at 12 months..$^{10}$ On muscle strength and pulmonary function, the trials found no significant difference between deflazacort and prednisone at 52 weeks.

In addition to the RCTs, we identified 3 observational studies that evaluated loss of ambulation in patients on deflazacort and prednisone. ${ }^{11-13}$ Two of the studies found that treatment with deflazacort significantly delayed loss of ambulation by around 3 years compared with prednisone. ${ }^{11,13}$ The third study found very little difference in mean age at loss of ambulation with either short-term (4 months to 3 years) or long-term (greater than 3 years) deflazacort or prednisone use, although the study was not designed primarily to compare the 2 treatments. ${ }^{12}$

The difference in chemical structure between deflazacort and prednisone suggest that there could be differences in tolerability between the 2 drugs. Our review of the RCTs and longterm observational studies showed that deflazacort-treated patients appeared to have less unwanted weight gain and fewer weight-related adverse events (AEs) than those treated with prednisone, while cataract formation, reduction in growth, and risk of fracture appeared to be greater with deflazacort. However, evidence on other important harms (e.g., behavior change) is inadequate to come to definite conclusions and overall does not appear to clearly favor either deflazacort or prednisone.

Eteplirsen and Golodirsen Versus Supportive Care. For eteplirsen, we identified 1 phase $2 \mathrm{~b}$ placebo-controlled trial with 12 patients and 3 open-label trials. ${ }^{14-16}$ For golodirsen, we identified 1 ongoing 2-part phase $1 / 2$ trial of golodirsen with part 1 as a placebo-controlled, dose escalation trial of 12 patients, and part 2 as an open-label extension of 25 patients. ${ }^{14}$ Participants in the eteplirsen and golodirsen trials had to have stable respiratory function and were all on a stable dose of corticosteroids. The main efficacy outcome in these trials was increase in the level of dystrophin-positive fibers on muscle biopsy. In the eteplirsen RCT, patients in the $30 \mathrm{mg} / \mathrm{kg}$ eteplirsen arm had very little change in absolute dystrophin levels, with dystrophin levels remaining less than $1 \%$ of normal in all patients after 180 weeks of treatment. However, eteplirsen did produce a positive relative change from baseline after 24 weeks compared with the placebo group: mean change in percent of dystrophin (23\% vs. $-4 \%$, mean difference $=27 \%$, $P \leq 0.002$ ). Similar results were seen with golodirsen, with an increase of absolute mean dystrophin levels of $0.92 \%$ to just over $1 \%$ of normal in patients treated for 48 weeks. Functional outcome reported in the eteplirsen trial showed that patients on $30 \mathrm{mg} / \mathrm{kg}$ of eteplirsen experienced a greater loss of distance walked in a 6-minute walk test (6MWT) compared with those on placebo ( -128 meters vs. -26 meters). However, the investigators attributed the larger decline in the $30 \mathrm{mg} / \mathrm{kg}$ arm to 2 of the 4 patients who had rapidly progressive disease. Observational data comparing open label eteplirsen with matched or historical controls suggest that treated patients may experience improvements in motor function (162 meters less decline in 6MWT) and pulmonary function (2\%-4\% slower decline in annual percent predicted forced vital capacity) by the fourth year. ${ }^{15-17}$ There are currently no functional outcome data on golodirsen.

Harms of the exon-skipping therapies appear to be limited. The majority of AEs observed in the trials were mild to moderate and included procedural pain, incision site hemorrhage or hematoma, hypokalemia, vomiting, balance disorder, headache, pyrexia, and contact dermatitis. There were no AEs leading to discontinuation, and no deaths were reported.

\section{Limitations of the Clinical Evidence}

As is common with treatments for rare disease, the evidence base for this review is limited by the few RCTs, small patient populations, and lack of long-term efficacy data. Although observational data have provided longer-term follow-up on deflazacort versus prednisone, the uncertainty about the natural history of the disease, lack of consistent dosing, differences in outcomes measures, and potential selection bias of patients who could afford to buy deflazacort out-of-pocket from overseas before its approval in the United States makes the interpretation of the observational evidence difficult. Additionally, data for exon-skipping therapies consist primarily of surrogate outcomes (e.g., dystrophin levels), and the threshold for dystrophin expression that results in meaningful clinical improvement has not yet been defined. Furthermore, there is limited or no evidence demonstrating improvements in function, and the evidence that exists using comparison with historical controls is vulnerable to significant selection and effort bias. Thus, as even the FDA has acknowledged, the clinical efficacy of exonskipping therapies is still unclear.

\section{Long-Term Cost-Effectiveness}

We evaluated the lifetime cost-effectiveness of all treatments using a de novo 5-state partitioned survival model informed by key clinical trials, cohort studies, and previous relevant economic modeling in DMD. The 5 health states in the model were early ambulatory, late ambulatory, early nonambulatory, late nonambulatory, and death. The model was developed with 2 base cases under ICER's ultra-rare disease value frameworka health care sector perspective and a societal perspective. The base-case model was used to project total costs, life-years, and quality-adjusted life-years (QALYs) over a lifetime time horizon, as well as to determine threshold treatment effects. Costs and outcomes were discounted at 3\% per year. The model used a hypothetical cohort of patients with DMD who began treatment at the age of 5 years. Patients were partitioned into relevant health states based on a previous comprehensive 


\begin{tabular}{|c|c|c|c|c|c|}
\hline TABL & \multicolumn{5}{|c|}{$\begin{array}{l}\text { Base-Case Results from Health Care } \\
\text { Sector and Modified Societal Perspective } \\
\text { for Prednisone with Supportive Care } \\
\text { and Deflazacort with Supportive Care }\end{array}$} \\
\hline & \multicolumn{3}{|c|}{ Discounted } & \multicolumn{2}{|c|}{$\begin{array}{l}\text { Deflazacort Versus } \\
\text { Prednisone }\end{array}$} \\
\hline & $\begin{array}{c}\text { Total Cost } \\
\$\end{array}$ & QALYs & LYs & $\begin{array}{l}\text { Cost per } \\
\text { QALY } \\
\text { Gained, \$ }\end{array}$ & $\begin{array}{l}\text { Cost per } \\
\text { LY Gained } \\
\$\end{array}$ \\
\hline \multicolumn{6}{|c|}{ Health sector perspective } \\
\hline Prednisone ${ }^{a}$ & 464,000 & 6.88 & 15.05 & \multirow{2}{*}{344,000} & \multirow{2}{*}{361,000} \\
\hline Deflazacort ${ }^{\mathrm{a}}$ & $1,010,000$ & 8.40 & 16.64 & & \\
\hline \multicolumn{6}{|c|}{ Modified societal perspective } \\
\hline Prednisone ${ }^{a}$ & $1,240,000$ & 6.88 & 15.05 & \multirow{2}{*}{371,000} & \multirow{2}{*}{390,000} \\
\hline Deflazacort ${ }^{\mathrm{a}}$ & $1,830,000$ & 8.40 & 16.64 & & \\
\hline
\end{tabular}

analysis of international clinical trial data involving steroid treatment for DMD. Clinical input on corticosteroids for the model was obtained from the observational study that had the largest evidenced-based treatment effect for deflazacort. ${ }^{11}$ Full details on ICER's cost-effectiveness analysis and model are available on ICER's website at https://icer-review.org/material/ dmd-final-evidence-report/.

Deflazacort Versus Prednisone. For deflazacort versus prednisone, because of limitations in the clinical evidence, we used extremely favorable assumptions regarding its treatment effects. As such, our analyses reflect a lower bound for its expected cost-effectiveness relative to prednisone plus supportive care. Our base-case results showed that the lower bound incremental cost-effectiveness ratio of deflazacort at a net annual price of $\$ 81,400$ was $\$ 361,000$ per QALY, well above the range of commonly accepted thresholds of $\$ 50,000$ $\$ 150,000$ per QALY, despite highly favorable assumptions about treatment effects (Table 1). Similar results were seen for the cost per life-year gained.

Eteplirsen and Golodirsen Versus Supportive Care. For eteplirsen and golodirsen, as previously noted, we judged that there was insufficient evidence to guide assumptions about the magnitude of beneficial treatment effects. However, given that the price for eteplirsen is available, we were able to perform threshold analyses to determine how effective the treatment would need to be in order to achieve different levels of costeffectiveness. Even under the extreme threshold assumption that eteplirsen restores all patients with DMD to perfect health for an additional 40 years of life, at the current annual cost of $\$ 1,002,000$, the cost per QALY was calculated to be $\$ 1,110,000$ and cost per life-year gained was $\$ 1,450,000$, far exceeding commonly accepted thresholds for cost-effectiveness. If one assumes that golodirsen will have the same costs as eteplirsen,

\section{TABLE 2 Other Benefits or Disadvantages: Deflazacort Versus Prednisone}

Is it likely that treatment with deflazacort offers one or more of the following potential "other benefits" that are not adequately captured in the base-case cost-effectiveness model? a

Compared with prednisone, deflazacort will reduce important $1 / 17$ health disparities across racial, ethnic, gender, socioeconomic, or regional categories.

Compared with prednisone, deflazacort will significantly reduce caregiver or broader family burden.

There are other important benefits or disadvantages that should have an important role in judgments of the long-term value for money of deflazacort.

aVotes were taken on an abbreviated list of potential other benefits, since reduced complexity, a novel mechanism of action approach, and improving productivity were determined not to apply to deflazacort.

then the threshold analyses would be the same for golodirsen as for eteplirsen.

\section{Limitations of the Cost-Effectiveness Model}

The current limited evidence available on the natural history and treatment of DMD only allowed for 5 health states in the model. In addition, evidence for the effect of deflazacort versus prednisone was limited and mixed. As such, our basecase analysis focused on an assumption of the most favorable treatment effect for deflazacort obtained from an observational study that itself may have been subject to selection bias. Finally, as previously described, since there was insufficient evidence of a treatment effect for eteplirsen, we could only report threshold analyses.

\section{Policy Discussion}

The New England Comparative Effectiveness Public Advisory Council (New England CEPAC, https://icer-review.org/programs/new-england-cepac/) is one of the independent appraisal committees convened by ICER to engage in public deliberation of the evidence on clinical effectiveness and cost-effectiveness of health care interventions. The New England CEPAC is composed of medical evidence experts, including practicing clinicians, methodologists, and leaders in patient engagement and advocacy. Their deliberation includes input from clinical experts and patient representatives specific to the condition under review, as well as formal comment from manufacturers and the public. A policy roundtable concludes each meeting during which representatives from insurers and manufacturers join clinical experts and patient representatives to discuss how best to apply the findings of the evidence to clinical practice, insurance coverage, and pricing negotiations.

The ICER report on treatments for DMD was the subject of a New England CEPAC meeting in July 2019. Following discussion, the CEPAC panel members voted 10-7 that the clinical evidence was adequate to demonstrate greater net health 


\section{TABLE 3 Other Benefits or Disadvantages: Eteplirsen or Golodirsen}

Is it likely that treatment with eteplirsen or golodirsen offers one or more of the following potential "other benefits" that are not adequately captured in the base-case cost-effectiveness model? a

Eteplirsen and golodirsen will reduce important health

disparities across racial, ethnic, gender, socioeconomic, or regional categories.

Eteplirsen and golodirsen will significantly reduce caregiver or broader family burden.

Eteplirsen and golodirsen will have a significant impact on improving patients' ability to return to work and/or their overall productivity.

There are other important benefits or disadvantages that should have an important role in judgments of the value of eteplirsen and golodirsen.

aVotes were taken on an abbreviated list of potential other benefits, since reduced complexity and a novel mechanism of action approach were determined not to apply to eteplirsen or golodirsen.

benefits of deflazacort compared with prednisone. The panel noted that RCT data were suggestive of some marginal benefit and that 1 large observational trial suggested an even greater relative benefit in ambulation. In contrast, for the exon-skipping drugs, the panel voted that the evidence was not adequate to judge the net health benefits provided by adding eteplirsen (16-1) or golodirsen (17-0) to corticosteroids and supportive care alone.

Based on ICER's adaptation of the value framework for rare diseases, treatments for DMD may have a number of potential other benefits and contextual considerations. The CEPAC panel voted separately on these "other potential benefits" and "contextual considerations" as part of a process intended to signal to policymakers that there may be important considerations when making judgments about long-term value for money that are not adequately captured in analyses of clinical effectiveness and/or cost-effectiveness. The results of these votes are shown in Tables 2-5 and serve to highlight several factors that the panel felt were particularly important for judgments of value.

The culminating vote of the CEPAC panel, intended to reflect its integration of the relevant elements of the value assessment framework, was on the "long-term value for money" of deflazacort and eteplirsen. The strong majority (14/17) of panel members voted that the long-term value for money of deflazacort versus prednisone is "low." For eteplirsen, the panel vote also was largely for "low" overall long-term value for money at current pricing (16/17).

The policy roundtable discussion explored how best to translate the evidence and broader perspectives discussed into clinical practice and into pricing and insurance coverage policies. The full set of policy recommendations can be found in the final evidence report on the ICER website at

\section{TABLE 4 Contextual Considerations: Deflazacort}

Are any of the following contextual considerations important in assessing deflazacort's long-term value for money? ${ }^{a}$

Deflazacort is intended for the care of individuals with a condition of particularly high severity in terms of impact on length of life and/or quality of life.

Deflazacort is intended for the care of individuals with a condition that represents a particularly high lifetime burden of illness.

There is significant uncertainty about the long-term risk of serious side effects of deflazacort.

There is significant uncertainty about the magnitude or durability of the long-term benefits of deflazacort.

There are additional contextual considerations that should have an important role in judgments of the value of deflazacort.

aVotes were taken on an abbreviated list of contextual considerations, since deflazacort was determined not to be the first to offer any improvement for DMD. DMD = Duchenne muscular dystrophy.

https://icer-review.org/material/dmd-final-evidence-report/. The key policy recommendations are as follows:

1. To balance early access with the need for fair pricing and ongoing evidence development, drugs such as eteplirsen and golodirsen granted accelerated approval should be priced closer to the marginal cost of production until clinical benefits are proven.

2. Patient groups and clinicians should work with manufacturers early in the design of clinical trials to embed the expectation that patient-centered outcomes will be measured in key trials and that the company will bring an effective drug to market at a price that aligns fairly with the demonstrated benefits for patients.

3. Given the high cost and substantial remaining uncertainty regarding the benefits of these treatments in certain subpopulations, it is reasonable for insurers and other payers to develop prior authorization criteria to ensure prudent use. Prior authorization criteria should be based on clinical evidence, specialty society guidelines, and input from clinical experts and patient groups. The process for authorization should be clear and efficient for providers. Considerations for prior authorization include the following:

o Diagnosis: Payers may reasonably require submission of genetic analysis (which would have been routinely performed as part of diagnosis) for the use of deflazacort and genetic analysis demonstrating DMD with a mutation amenable to exon 51 skipping or exon 53 skipping for the exon-skipping therapies.

o Step therapy: For deflazacort, failure of prednisone is based on toxicity, so a step therapy policy should not require a fixed period of time for a trial of prednisone but rather documentation of adverse effects.

o Dosage restrictions: Clinical experts stated that they generally only use the approved dose of deflazacort (0.9 $\mathrm{mg} / \mathrm{kg} /$ day). For eteplirsen, at its labeled dosing for 


\section{TABLE 5}

Contextual Considerations: Eteplirsen and Golodirsen

\begin{tabular}{l|c}
$\begin{array}{l}\text { Are any of the following contextual considerations important in assessing } \\
\text { eteplirsen and golodirsen's long-term value for money? }\end{array}$ \\
$\begin{array}{l}\text { Eteplirsen and golodirsen are intended for the care of } \\
\text { individuals with a condition of particularly high severity in } \\
\text { terms of impact on length of life and/or quality of life. }\end{array}$ \\
$\begin{array}{l}\text { Eteplirsen and golodirsen are intended for the care of } \\
\text { individuals with a condition that represents a particularly high } \\
\text { lifetime burden of illness. }\end{array}$ \\
$\begin{array}{l}\text { Eteplirsen and golodirsen are the first to offer any improvement } \\
\text { for patients with this condition. }\end{array}$ & $15 / 17$ \\
$\begin{array}{l}\text { There is significant uncertainty about the long-term risk of } \\
\text { serious side effects of eteplirsen and golodirsen. }\end{array}$ & $8 / 17$ \\
$\begin{array}{l}\text { There is significant uncertainty about the magnitude or } \\
\text { durability of the long-term benefits of eteplirsen and golodirsen. }\end{array}$ & $11 / 17$ \\
$\begin{array}{l}\text { There are additional contextual considerations that should have } \\
\text { an important role in judgments of the value of eteplirsen and } \\
\text { golodirsen. }\end{array}$ & $16 / 17$ \\
\hline
\end{tabular}

typical weight patients, despite initial statements from the manufacturer suggesting much lower costs, it can be expected to cost approximately $\$ 1$ million per year. Until evidence from clinical trials demonstrates added benefit from higher doses, it is reasonable to restrict coverage to the dose listed in the FDA-approved labeling.

o Renewal criteria: There is no reason to require renewal criteria demonstrating the attestation of benefit for continuing deflazacort or exon-skipping therapies, since continued clinical decline is expected on treatment.

\section{Conclusions}

Current evidence demonstrates comparable or perhaps better clinical benefit of deflazacort versus prednisone. However, at current pricing in the U.S. market, incorporating the most highly favorable assumptions around treatment effects, the resulting incremental cost-effectiveness ratio for deflazacort exceeds commonly cited cost-effectiveness thresholds. For the exon-skipping therapies eteplirsen and golodirsen, we have insufficient evidence to judge clinical benefit in the absence of adequate studies examining patient-centered outcomes. Furthermore, at the current price, our analyses showed that eteplirsen (and golodirsen if priced similarly) could never be cost-effective at commonly cited cost-effectiveness thresholds, even assuming extreme curative treatment effects that are not consistent with existing data and clinical experience. Thus, deflazacort and eteplirsen were judged to represent low long-term value for the money. Although treatment advances for DMD are desperately needed, further efforts are required to ensure that existing therapies are effective, that patientcentered outcomes are measured and reported in clinical trials, and that the price of treatments aligns fairly with the benefits to patients before the widespread use of new therapies.

\section{Authors}

FOLUSO AGBOOLA, MBBS, MPH; NOEMI FLUETSCH, MPH; DAVID M. RIND, MD, MSc; and STEVEN D. PEARSON, MD, MSc, Institute for Clinical and Economic Review, Boston, Massachusetts. GRACE A. LIN, MD, MAS, Department of Medicine and Philip R Lee Institute for Health Policy Studies, University of California, San Francisco, and SURREY M. WALTON, PhD, Center for Pharmacoepidemiology and Pharmacoeconomic Research, University of Illinois at Chicago.

AUTHOR CORRESPONDENCE: Foluso Agboola, MBBS, MPH, Institute for Clinical and Economic Review, Two Liberty Square, 9th Fl., Boston, MA 02109. E-mail: fagboola@icer-review.org.

\section{DISCLOSURES}

Funding for this summary was contributed by Arnold Ventures, Commonwealth Fund, California Health Care Foundation, National Institute for Health Care Management (NIHCM), New England States Consortium Systems Organization, Blue Cross Blue Shield of Massachusetts, Harvard Pilgrim Health Care, Kaiser Foundation Health Plan, and Partners HealthCare to the Institute for Clinical and Economic Review (ICER), an independent organization that evaluates the evidence on the value of health care interventions. ICER's annual policy summit is supported by dues from Aetna, America's Health Insurance Plans, Anthem, Allergan, Alnylam, AstraZeneca, Biogen, Blue Shield of CA, Cambia Health Services, CVS, Editas, Express Scripts, Genentech/Roche, GlaxoSmithKline, Harvard Pilgrim, Health Care Service Corporation, Health Partners, Johnson \& Johnson (Janssen), Kaiser Permanente, LEO Pharma, Mallinckrodt, Merck, Novartis, National Pharmaceutical Council, Premera, Prime Therapeutics, Regeneron, Sanofi, Spark Therapeutics, and United Healthcare.

Agboola, Fluetsch, Rind, and Pearson are employed by ICER. Lin reports support from ICER during work on this economic model and grants from Mount Zion Health Fund, National Institutes of Health (National Cancer Institute and National Heart, Lung, and Blood Institute), and the TobaccoRelated Diseases Research Program, unrelated to this work. Walton reports support from ICER for work on this economic model and unrelated consulting fees from Baxter.

\section{REFERENCES}

1. Hoffman EP, Fischbeck KH, Brown RH, et al. Characterization of dystrophin in muscle-biopsy specimens from patients with Duchenne's or Becker's muscular dystrophy. N Engl J Med. 1988;318(21):1363-68.

2. Mendell JR, Shilling C, Leslie ND, et al. Evidence-based path to newborn screening for Duchenne muscular dystrophy. Ann Neurol. 2012;71(3):304-13.

3. Ciafaloni E, Fox DJ, Pandya S, et al. Delayed diagnosis in duchenne muscular dystrophy: data from the Muscular Dystrophy Surveillance, Tracking, and Research Network (MD STARnet). J Pediatr. 2009;155(3):380-85.

4. Humbertclaude V, Hamroun D, Bezzou K, et al. Motor and respiratory heterogeneity in Duchenne patients: implication for clinical trials. Eur J Paediatr Neurol. 2012;16(2):149-60.

5. Calvert LD, McKeever TM, Kinnear WJ, Britton JR. Trends in survival from muscular dystrophy in England and Wales and impact on respiratory services. Respir Med. 2006;100(6):1058-63.

6. Steffensen B, Otto C, Werlauff U, et al. Health related quality of life in European adults with DMD: results from the Care-NMD-project [abstract] Neuromuscul Disord. 2015;25:S302. Available at: https://www.nmd-journal. com/article/S0960-8966(15)00593-3/abstract. Accessed March 11, 2020. 
7. Lin G, Walton S, Agboola F, et al. Deflazacort, eteplirsen, and golodirsen for Duchenne muscular dystrophy: effectiveness and value. Final evidence report. Institute for Clinical and Economic Review. August 15, 2019. Available at: https://icer-review.org/wp-content/uploads/2018/12/ICER_ DMD-Final-Report_081519.pdf. Accessed March 11, 2020.

8. Griggs RC, Miller JP, Greenberg CR, et al. Efficacy and safety of deflazacort vs prednisone and placebo for Duchenne muscular dystrophy. Neurology. 2016;87(20):2123-31.

9. Karimzadeh P, Ghazavi A. Comparison of deflazacort and prednisone in Duchenne muscular dystrophy. Iran J Child Neurol. 2012;6(1):5-12. Available at: https://www.researchgate.net/publication/277034512_Comparison_of_ Deflazacort_and_Prednisone_in_Duchenne_Muscular_Dystrophy. Accessed March 11, 2020.

10. Bonifati MD, Ruzza G, Bonometto P, et al. A multicenter, double-blind, randomized trial of deflazacort versus prednisone in Duchenne muscular dystrophy. Muscle Nerve. 2000;23(9):1344-47.

11. McDonald CM, Henricson EK, Abresch RT, et al. Long-term effects of glucocorticoids on function, quality of life, and survival in patients with Duchenne muscular dystrophy: a prospective cohort study. Lancet. 2018;391(10119):451-61.
12. Kim S, Campbell KA, Fox DJ, Matthews DJ, Valdez R. Corticosteroid treatments in males with Duchenne muscular dystrophy: treatment duration and time to loss of ambulation. J Child Neurol. 2015;30(10):1275-80.

13. Bello L, Gordish-Dressman H, Morgenroth LP, et al. Prednisone/prednisolone and deflazacort regimens in the CINRG Duchenne Natural History Study. Neurology. 2015;85(12):1048-55.

14. Mendell JR, Rodino-Klapac LR, Sahenk Z, et al. Eteplirsen for the treatment of Duchenne muscular dystrophy. Ann Neurol. 2013;74(5):637-47.

15. Mendell JR, Goemans N, Lowes LP, et al. Longitudinal effect of eteplirsen versus historical control on ambulation in Duchenne muscular dystrophy. Ann Neurol. 2016;79(2):257-71.

16. Khan N, Eliopoulos H, Han L, et al. Eteplirsen treatment attenuates respiratory decline in ambulatory and non-ambulatory patients with Duchenne muscular dystrophy. J Neuromuscul Dis. 2019;6(2):213-25.

17. Mendell J, Goemans N, Rodino-Klapac L, et al. Eteplirsen, a phosphorodiamidate morpholino oligomer for Duchenne muscular dystrophy: longitudinal comparison to external controls on 6-minute walk test and loss of ambulation [abstract]. Neurology. 2017;88 (16 Suppl):S42.004. Available at: https://n.neurology.org/content/88/16_Supplement/S42.004. Accessed March 11, 2020.

\title{
Evaluating a Stone of Hope: ICER's 2019 Review of Treatments for Duchenne Muscular Dystrophy
}

\author{
John Frederick Brandsema, MD
}

\section{COMMENTARY}

(DMD) is a severe X-linked neurodegenerative disorder that is the most common muscular dystrophy of childhood, affecting about 1 in 3,500 male births ${ }^{1}$; at a large pediatric neuromuscular clinic, the diagnosis may be disclosed to multiple families per month. Typically, this conversation occurs with the family of a young boy around 3 or 4 years of age. The discussion of natural history

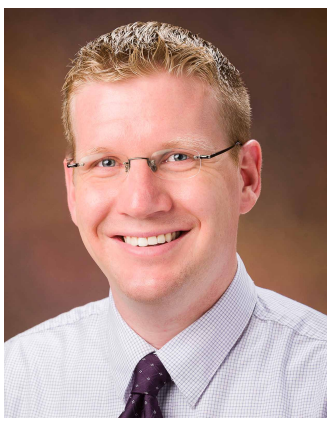

forms of dystrophinopathy, such as the Becker form defined by ambulation beyond age $16 .^{1}$

Before 2016, therapeutic options included optimized interdisciplinary supportive care as well as corticosteroids, the combination of which may increase lifespan into the fourth or rarely fifth decade of life for a larger proportion of patients. Steroids tend to slow but not reverse the relentless decline in muscle strength and are the standard of care in consensus guidelines. The typical effect outlines likely prognostic milestones such as loss of ambulation before age 13, development of detectable and progressive cardiomyopathy and respiratory failure in school age years, and death in the late second or early third decade of life. ${ }^{1}$ Cognition does not decline, but a significant proportion of patients may have cognitive symptoms ranging from behavioral issues such as anxiety or attention deficit hyperactivity disorder to more severe cognitive impairment and autism. There are also milder is around 1-3 years slower disease milestones, but this comes with a host of iatrogenic comorbidities, most prominently increased risk for behavioral issues, linear growth suppression and obesity in younger patients, and eventually poorer bone health with risk for cataracts in older patients. ${ }^{1}$

Treatment options include prednisone/prednisolone versus deflazacort: The ideal steroid type and schedule is still not firmly established since dose reductions due to side effects or family preference, alternative dosing schedules (e.g., daily, weekend-only, and 10 days on and 10 days off), and heterogeneity in the disease phenotype among patients make even matched cohort comparisons have limitations. ${ }^{2}$ 